\title{
Learning Effectiveness Assessment between Primary School Students and Adults in Basic Life Support Education
}

\author{
Ming-Fang Wang, ${ }^{1}$ Yi-Kan Wu, ${ }^{1}$ Cheng-Yu Chien $\mathbb{D}^{1},{ }^{1,2,3}$ Li-Heng Tsai, ${ }^{1}$ Chen-Bin Chen, \\ Chen-June Seak $\mathbb{1},{ }^{1}$ Chi-Chun Lin, ${ }^{1,2}$ Chien-Hsiung Huang, ${ }^{1,4}$ Chung-Hsien Chaou $\mathbb{1}{ }^{1}{ }^{1}$ \\ Hsiao-Jung Tseng, ${ }^{5}$ and Chip-Jin $\mathrm{Ng}{ }^{1}{ }^{1}$ \\ ${ }^{1}$ Department of Emergency Medicine, Chang Gung Memorial Hospital, Linkou and College of Medicine, Chang Gung University, \\ Tao-Yuan, Taiwan \\ ${ }^{2}$ Department of Emergency Medicine, Ton-Yen General Hospital, Zhubei, Taiwan \\ ${ }^{3}$ Graduate Institute of Business and Management, Chang Gung University, Taoyuan, Taiwan \\ ${ }^{4}$ Department of Emergency Medicine, New Taipei City Hospital, New Taipei City, Taiwan \\ ${ }^{5}$ Biostatistical Unit, Clinical Trial Center, Chang Gung Memorial Hospital, Linkou, Taiwan \\ Correspondence should be addressed to Chip-Jin Ng; ngowl@ms3.hinet.net
}

Received 21 January 2021; Revised 7 February 2021; Accepted 12 February 2021; Published 24 February 2021

Academic Editor: Yan-Ren Lin

Copyright (c) 2021 Ming-Fang Wang et al. This is an open access article distributed under the Creative Commons Attribution License, which permits unrestricted use, distribution, and reproduction in any medium, provided the original work is properly cited.

\begin{abstract}
Background. Out-of-hospital cardiac arrest (OHCA) remains a big issue of critical care. It is well known that bystander cardiopulmonary resuscitation (CPR) with an automated external defibrillator (AED) used did improve the survival rate. Therefore, CPR education including basic life support (BLS) and AED has been advocated for years. It showed significant improvement of knowledge and willingness to perform CPR through adolescents after the course. However, little is known regarding the ability and learning effectiveness of school students who attend such courses. Therefore, this study aimed to evaluate the CPR effectiveness of both adolescents (12 years old) and adults who undergo the same course of BLS and AED. Methods. This is a retrospective study. Sixth-grade elementary school students in Northern Taiwan were selected to compare with the adult group. Both took 90 minutes of the BLS and AED course by the doctor with BLS instructor qualification. The primary outcomes were CPR quality and passing or failing the skill examination parameters. The secondary outcome was the posttraining written test and questionnaire of CPR willingness. Results. In the written test, there was a statistical difference in the pretest score except AED knowledge, but no difference was revealed in the posttest score. No statistical difference in CPR quality was noted. In the skill examination, only checking breathing status had statistical difference (elementary group (71\%) vs. adult group (86\%) $(p=0.003)$ ). Conclusion. We revealed that sixth-grade elementary students' performance in CPR and AED was similar to that of adults after completing the current 90-minute course. Therefore, we strongly advocate offering CPR and AED courses to 12-year-old children, and these courses should emphasize checking the victim's breathing status.
\end{abstract}

\section{Introduction}

Out-of-hospital cardiac arrest (OHCA) is a critical public health concern. OHCA has a lower incidence rate compared with other diseases; however, it has a high mortality rate. In the United States, the OHCA rate per 100,000 people is 3.5 [1]. Between 2000 and 2012 in Taiwan, approximately 51.1 people per 100,000 experienced OHCA [2]. According to a study conducted in Paris, up to $70 \%$ of OHCAs occur in residential areas, and 30\% occur in public areas [3]. Moreover, a recent retrospective study in Taiwan with data from 2012 to 2016 revealed that $80 \%$ of OHCAs occur in private places [4]. A systematic review also reported that approximately $53 \%$ of events are witnessed by a bystander, and bystander cardiopulmonary resuscitation (CPR) remains low at $32 \%$ [5]. However, bystander CPR rates vary 
considerably worldwide, ranging from $10 \%$ to $65 \%$ in the United States [6]. Multiple factors are responsible for these large differences in the bystander CPR rate, including social economic status, racial and educational characteristics, and location of the collapse [7-9].

Bystander CPR with an automated external defibrillator (AED) is widely known to improve the survival rate. Moreover, bystander CPR significantly increases (by up to two- to four-fold) 30-day and 1-year survival regardless of witnessed status [10]. Nevertheless, according to related studies in Taiwan, bystander CPR rates before the arrival of an emergency medical technician (EMT) ranged from $17 \%$ in 2008 to approximately $30 \%$ between 2012 and $2016[4,11]$. According to a US study, bystander CPR rates have increased slightly over time: from $28.2 \%$ in $2005-2006$ to $36.3 \%$ in 2012 [12]. CPR education has been expanding for years, with considerable public health benefits; however, obstacles remain regarding the execution of $\mathrm{CPR}$ by the public. According to a study conducted in Taiwan, the main reasons people hesitate to perform CPR are fear of legal consequences (44\%) and harming patients (36.5\%) [13]. Concerning the legal aspect, the Good Samaritan Law was passed in 2013 in Taiwan to protect people against legal consequences if they perform CPR incorrectly on a stranger in a critical situation. In addition to legal protection, CPR educational training should also be promoted to increase people's willingness to perform CPR.

Currently, the American Heart Association (AHA) has a specific education program for adolescents. In Taiwan, CPR education has been provided for high-school students for years. To extend the benefits of such training, we started a new CPR training program in Taiwan for adults, and it revealed noninferior results to the conventional $\mathrm{CPR}$ training program [14]. However, little is known regarding the ability and learning effectiveness of school students who attend such basic life support (BLS) and AED courses. A European group named Kids Save Lives has claimed that training school children in CPR is highly effective, and 12 years is the suitable age to start teaching cardiac compression [15]. However, CPR quality is highly related to the body mass index (BMI) and exercise habits in EMTs [16]. Whether current adult CPR teaching programs are suitable for adolescents and whether these younger students can achieve the same CPR effectiveness as adults remain unknown. Therefore, the purpose of this study was (1) to evaluate the CPR effectiveness of both adolescents (12 years old) and adults who undergo the same course related to BLS and AED in the same environment and (2) to prove that the current adult BLS course is suitable for adolescents.

\section{Method}

2.1. Study Design, Setting, and Participants. This retrospective observation study was approved by the Chang Gung Memorial Foundation Institutional Review Board (approval number: 202000464B0). We extracted data from the database of an education program (IGOGO) and considered training courses between January 2018 and July 2018. The extracted data had to meet inclusion criteria, including students having the same training date and classroom, and participants were subsequently divided into an elementary sixth-grade students (elementary group) and adult group. The purpose of the training program was to promote the long-term implementation of CPR teaching combined with the use of AED for the public. To evaluate learning effectiveness in both elementary school students and adults, we selected participants who did not receive any CPR training for at least 1 year prior to taking this training course. Participants who were unable to kneel to perform CPR and those who were pregnant were excluded. A total of 308 participants were analyzed in the study, including sixthgrade students and teaching staff, security guards, and volunteers.

2.2. Education Course. IGOGO in Taiwan has been offering courses for many years. We use a standard 90-minute BLS training program, which is similar to the AHA course. The AHA program is a 90-minute, instructor-led, and classroom-based training program that employs the practicewhile-learning format. The learning content includes an introduction to relevant laws, the purpose of CPR and AED, chains of survival, demonstration of the adult BLS sequence, CPR with AED use, and hands-on compression-only adult CPR.

The IGOGO program is taught by emergency physicians with BLS instructor qualifications who are assisted by nurses and doctors. The ratio of participants to manikins to instructor is $8: 4: 1$. Sensor-equipped manikins (Resusci Anne with QCPR, Laerdal Medical AS, Norway) were used in the 2 -minute hands-on practice in both groups. Each course consisted of a 60-minute CPR teaching video with practice, 20 minutes of instruction related to AED operation, and a 10-minute discussion concerning the legal aspect of bystander CPR in Taiwan.

2.3. Data Collection. Data collection focused on training program-specific data and the demographic data of participants. We also obtained informed consent from all participants and removed any personally identifiable information. We compiled course-related information, including basic student and adult data (which contain age, weight, height, gender, previous exercise habits, whether there is any previous CPR learning experience, and when was the last learning experience), pretest and posttest (e.g., knowledge of CPR and AED) results, skill tests, and CPR willingness questionnaire, into a database [17] (Appendix 1 in Supplementary Materials). All questions in the written test were formulated by staff of the Taiwan Society of Emergency Medicine, Emergency Medical Services Department. We assessed learning effectiveness in several manners. We assessed CPR and related knowledge by using a written test, which contained 15 multiple-choice questions with a maximum score of 100 (Appendix 2 in Supplementary Materials). CPR performance was evaluated in two aspects: manikin feedback and examiner evaluation. Objective data, including compression depth, compression rate, and full chest recoil, were recorded and collected from the 


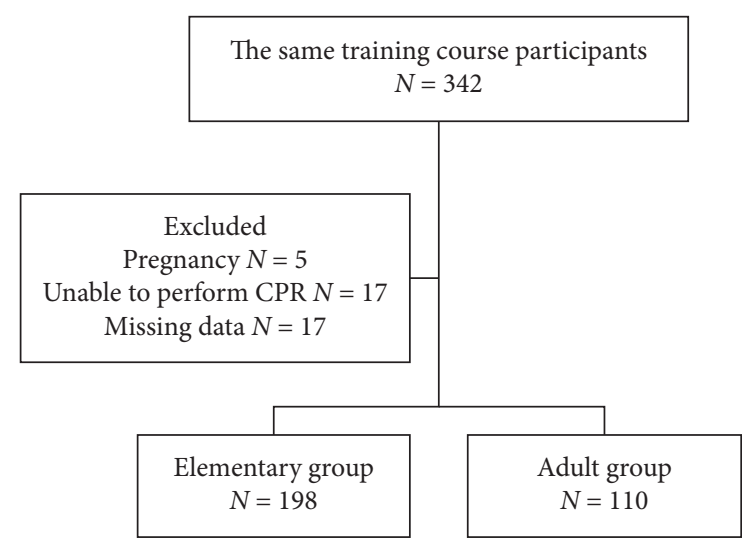

FIgURE 1: Flowchart. $N$ : number; CPR: cardiopulmonary resuscitation.

feedback manikin. We followed the updated 2015 AHA Guidelines for CPR and Emergency Cardiovascular Care, in which high-quality CPR is defined as follows: (1) a compression rate of $100-120$ beats per minute (bpm), (2) a compression depth of 5-6 cm, and (3) full chest wall recoil. Examiners rated participants' performance individually. Examiners assessed how well participants followed the BLS sequence in terms of skills on the checklist-from verifying scene safety to AED use (Appendix 3 in Supplementary Materials). We count each pass step as one point and fail step as zero points (total scores are 8). Ventilation was not included in this educational program because compressiononly CPR is the current recommendation.

2.4. Outcome Measures. The purpose of this study was to compare CPR effectiveness between elementary students and adults in the same setting (i.e., learning subject and environment). The primary outcomes were CPR quality (a compression rate of 100-120 bpm, a compression depth of $5-6 \mathrm{~cm}$, and full chest wall recoil) and passing or failing the following skill examination parameters: (1) confirm safety, (2) check consciousness, (3) call for help, (4) check breathing status, (5) CPR location, (6) CPR posture, (7) AED operation, and (8) AED pad location. The secondary outcome was the posttraining written test, questionnaire of CPR willingness, and total scores of skill examination (Appendices 1 and 2 in Supplementary Materials).

2.5. Statistical Analysis. Categorical variables were compared using the chi-squared test and are presented as numbers and percentages. Continuous variables are presented as means and standard deviations, and Student's $t$ test was used to compare the difference between two groups. The significance level $\alpha$ was set at 0.05 . The data were analyzed using IBM SPSS Statistics (version 25.0 for Windows; IBM Corp., Armonk, NY, USA).

\section{Results}

In total, 342 people participated in the training program including 210 students and 132 school staff (Figure 1). We
TABle 1: Demographic population.

\begin{tabular}{lcc}
\hline & Elementary group & Adult group \\
\hline Numbers & 198 & 110 \\
Age, years (SD) & $11.8(0.46)$ & $37.27(10.22)$ \\
Height, cm (SD) & $152.8(7.32)$ & $160.77(7.36)$ \\
Weight, kg (SD) & $41.9(8.70)$ & $60.75(11.43)$ \\
BMI (SD) & $17.83(3.018)$ & $23.85(3.357)$ \\
Female (\%) & $101(51.01 \%)$ & $76(69.09 \%)$ \\
Sport habits (\%) & $154(77.78 \%)$ & $51(46.36 \%)$ \\
$<1$ hour & $122(61.62 \%)$ & $28(25.45 \%)$ \\
$>1$ hour & $32(16.16 \%)$ & $23(20.91 \%)$ \\
CPR learning experience (\%) & $64(32.32 \%)$ & $94(85.45 \%)$ \\
$1 \sim 2$ years & $48(24.24 \%)$ & $72(65.45 \%)$ \\
$>2$ years & $7(3.54 \%)$ & $10(9.09 \%)$ \\
Unknown & $9(4.54 \%)$ & $12(10.91 \%)$ \\
Type of CPR (\%) & & \\
Hands-only CPR & $55(27.78 \%)$ & $71(64.54 \%)$ \\
\hline
\end{tabular}

Data are expressed as mean (SD) or $n$ (\%). cm: centimeter; kg: kilogram; SD: standard deviation; BMI: body mass index; CPR: cardiopulmonary resuscitation

TABle 2: Assessment of the cardiopulmonary resuscitation training course.

\begin{tabular}{lccc}
\hline & $\begin{array}{c}\text { Elementary } \\
\text { group }\end{array}$ & Adult group & $\begin{array}{c}p \\
\text { value }\end{array}$ \\
\hline $\begin{array}{l}\text { Post-written test score } \\
\text { (SD) }\end{array}$ & $89.77(8.28)$ & $91.62(8.68)$ & 0.064 \\
CPR (SD) & $91.75(12.00)$ & $\begin{array}{c}90.78 \\
(12.33)\end{array}$ & 0.499 \\
& & 79.09 & 0.872 \\
AED (SD) & $78.92(26.67)$ & $(27.78)$ & \\
Others (SD) & $92.61(11.66)$ & $92.18(13.57)$ & 0.77 \\
\hline Quality assessment & & & \\
BPM (SD) & $114.15(20.556)$ & 113.17 & 0.18 \\
Recoil (SD) & $75.7(32.0)$ & $77.2(31.0)$ & 0.33 \\
Depth (SD) & $4.68(0.95)$ & $5.22(0.81)$ & 0.12 \\
\hline
\end{tabular}

Data are expressed as mean (SD). SD: standard deviation; CPR: cardiopulmonary resuscitation; AED: automated external defibrillator; BPM: beats per minute.

excluded those who were pregnant, were unable to adequately perform CPR, or had incomplete information; thus, 308 participants were eligible for analysis. Among them, 198 were elementary school students and 110 were school staff. Table 1 lists the demographic statistics of the study. The mean age of elementary students was 11.8 years, and that of adults was 37.3 years. Females accounted for approximately half of the elementary group and $69 \%$ of the school staff. The two groups differed in terms of BMI, sport habits, and CPR learning experience.

The elementary group and adult group scored no difference in posttest (elementary group $=89.77$; adult group $=91.62 ; p=0.064$; Table 2 ). Regarding CPR quality, the elementary group achieved, on average, $114 \mathrm{bpm}$, a full chest recoil rate of $75.7 \%$, and a compression depth of $4.68 \mathrm{~cm}$. The adult group achieved, on average, $113 \mathrm{bpm}$, a full chest recoil rate of $77.2 \%$, and a compression depth of $5.22 \mathrm{~cm}$. In terms of CPR quality parameters, no significant 


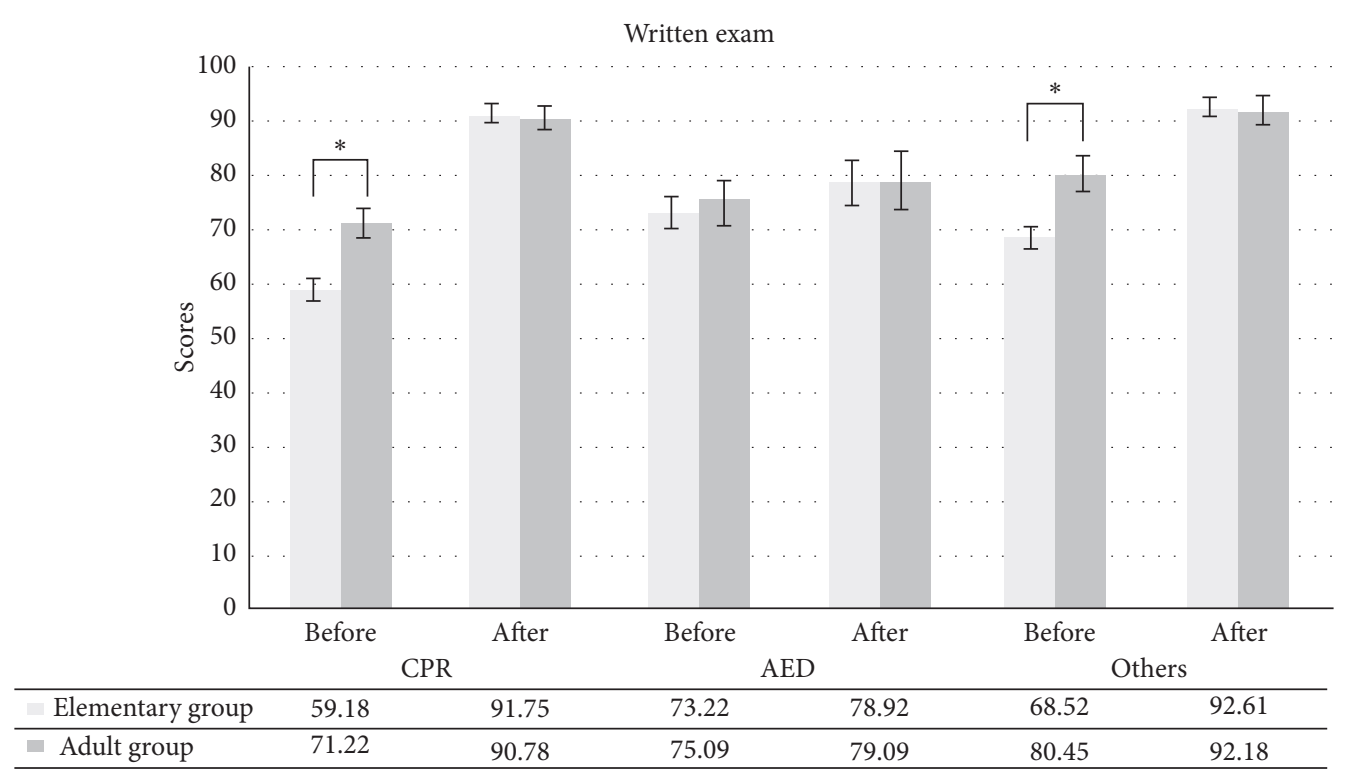

Figure 2: Difference of pre-/post-written exam after training. Data are expressed as score. ${ }^{*}$ indicates a significant difference. CPR: cardiopulmonary resuscitation; AED: automated external defibrillator.

TABLE 3: Skill examination parameters of cardiopulmonary resuscitation.

\begin{tabular}{lccc}
\hline & Elementary group & Adult group & $p$ value \\
\hline Numbers & 198 & 110 & \\
Confirm safety & $123(62.12 \%)$ & $72(65.07 \%)$ & 0.561 \\
Check consciousness & $172(86.87 \%)$ & $92(83.64 \%)$ & 0.437 \\
Call for help & $163(82.32 \%)$ & $94(85.45 \%)$ & 0.479 \\
Check breathing status & $142(71.72 \%)$ & $95(86.36 \%)$ & $0.003^{*}$ \\
CPR location & $154(77.78 \%)$ & $91(82.73 \%)$ & 0.302 \\
CPR posture & $153(77.27 \%)$ & $92(83.64 \%)$ & 0.185 \\
AED operation & $160(80.81 \%)$ & $95(86.36 \%)$ & 0.216 \\
AED pad location & $156(78.79 \%)$ & $96(87.27 \%)$ & 0.064 \\
Total scores & $6.18(1.284)$ & $6.61(1.342)$ & $0.006^{*}$ \\
\hline
\end{tabular}

Data are expressed as mean (SD) or $n(\%) .{ }^{*}$ indicates a significant difference. SD: standard deviation; CPR: cardiopulmonary resuscitation; AED: automated external defibrillator.

differences were observed between the two groups (Table 2). Figure 2 reveals a considerable improvement in both groups after taking the 90-minute course. The elementary and adult groups differed significantly in the pretest, except for AED knowledge. However, it showed no statistical difference in all three items (CPR, AED, and others) after the course.

Regarding skill items shown in Table 3, elementary students performed CPR as effectively as adults in almost all skills including verifying scene safety, checking consciousness, calling for help, CPR location, CPR posture, AED operation, and AED pad location. However, a significant difference was observed in checking breathing status and total scores: the success rate in the elementary group was $71 \%$, whereas it was $86 \%$ in the adult group $(p=0.003)$, and total scores were 6.18 (1.284) and 6.61 (1.342), respectively. We also investigated willingness to perform CPR. The results revealed no difference in willingness to perform hands-only $\mathrm{CPR}$ on an acquaintance but a significant difference in willingness to do so on a stranger (elementary group $=51 \%$; adult group $=39.1 \% ; p=0.045$; Table 4 ). The three major reasons why participants were unwilling to perform CPR on either an acquaintance or a stranger are fearing doing further harm, fearing performing CPR incorrectly, and being unwilling to perform cardiac compression.

\section{Discussion}

Our results revealed that, after undergoing the same training program, sixth-grade elementary students could perform CPR as effectively as adults could in three aspects, namely, compression depth, compression rate, and full chest recoil. However, elementary students struggled to meet the AHA high-quality benchmark for compression depth. In a study of school children aged 7-14 years, chest compression depth was highly correlated with children's age, weight, height, and BMI [18]. In a UK study that divided children into three groups, namely, 9-10, 11-12, and 13-14 years, only the 13-14year-old group could perform chest compression just as well as adults [19]. Therefore, when teaching high-performance CPR to elementary school students, instructors should focus on the knowledge and process, instead of requiring students to reach the depth mentioned in the AHA guidelines because of the children's limited ability to perform chest compression. Notably, the children and adults differed significantly in terms of checking breathing status despite following the same learning program and checklist; previous similar studies have not noted this disparity. Hence, instructors should devote special attention to teaching elementary students how to check breathing status in the future.

Currently, no regulations or rules stipulate when to implement CPR education in school settings in Taiwan. However, such programs are typically introduced in high school. According to the Kids Save Lives group in Europe, CPR training should start from the age of 12 years or even younger; moreover, annual CPR refresher courses should be 
TABLE 4: Willingness to perform CPR after training.

\begin{tabular}{lcc}
\hline & Elementary group & Adult group \\
\hline Willing to perform CPR on the associate & & $p$ value \\
Yes & $188(94.95 \%)$ & $11(10.0 \%)$ \\
No & & 5 \\
\hline Reasons of unwillingness & 10 & 7 \\
Afraid of doing further harm & 10 & 4 \\
Afraid of doing CPR incorrectly & 9 & 11 \\
Unwilling to perform cardiac compression & 7 & 2 \\
Afraid of legal issues & 4 & $43(39.09 \%)$ \\
Others & $101(51.01 \%)$ & $67(60.91 \%)$ \\
\hline Willing to perform CPR on a stranger & $97(48.99 \%)$ & 37 \\
Yes & & 28 \\
No & 43 & 24 \\
Reasons of unwillingness & 35 & 8 \\
Afraid of doing further harm & 31 & 21 \\
Afraid of doing CPR incorrectly & 27 & $0.045^{*}$ \\
Unwilling to perform cardiac compression & 23 & \\
Afraid of legal issues & & \\
Others & & \\
\hline
\end{tabular}

Data are expressed as $n(\%)$ or $n .{ }^{*}$ indicates a significant difference. CPR: cardiopulmonary resuscitation.

offered [20-22]. Training can be offered successfully with a low-cost manikin and equipment by either medical professionals or educated teachers [23]. Studies have reported that children aged 10-12 years have the same CPR effectiveness as adults do $[22,24]$. Our results corroborate those findings. In the written test, both children and adults exhibited considerable improvement between the pretest and posttest. Moreover, the 12-year-old elementary students had sufficient ability to undergo current CPR education and could comprehend and apply the training as well as adults could. Notably, AED knowledge in the pretest did not differ significantly between the two groups. This finding may be attributed to the widespread public-service announcements related to AED and also the implementation of AED in the school where our study was conducted. Therefore, as AED installations become more common, both promotion of AED and people's knowledge of it will increase.

Children included in this study could complete the CPR checklist items just as well as adults for almost all elements. However, children's performance in checking breathing status was inferior to that of adults; they either forget this element or performed it incorrectly. Checking breathing status is also a difficult task for adults: in conventional CPR education, adults struggle to differentiate bradypnea or agonal breathing. Thus, challenges faced by adolescents in CPR education might be understandable. A systematic review revealed that, by the age of 11-12 years, children can establish whether a victim is conscious and breathing normally [25]. We suggest to use virtual reality, augmented reality, and gamified learning to enhance the effectiveness of learning in the education program [26]. Results for AED pad location in the current study also merit further attention. Although the two groups did not differ significantly, the numerical difference was quite large $(71.72 \%$ vs. $86.36 \%)$. Thus, children may easily confuse the correct placement of an AED. For CPR education to have the same effectiveness in both children and adults, we argue that such programs should focus more on checking breathing status and AED pad location. More crucially, children should be taught to activate the local emergency medical services system earlier because most cardiac arrest cases occur in residential areas $[3,4]$.

Whether children can perform CPR adequately still requires further study. In a study in a rural area of Taiwan, a 50-minute CPR/AED course significantly improved the knowledge of adolescents and empowered them to be willing to perform CPR if necessary [27]. Our study revealed the same trend. Children seem to be more willing to perform $\mathrm{CPR}$ on either a stranger or an acquaintance. Therefore, early promotion of CPR education is valuable for elementary school students. As a result of such training, the bystander CPR rate might increase in the future. In addition, in our questionnaire, children reported anxiety regarding performing CPR on strangers mostly because they fear causing harm if they perform it incorrectly; adults were more concerned with the legal aspect and contracting an infectious disease $[28,29]$. Such reluctance may be overcome with sufficient training and by boosting children's confidence.

To sum up, we advocate offering the current 90-minute BLS and AED course to sixth-grade elementary students because they can understand the content and perform CPR as effectively as adults can. More widespread training may increase bystander CPR rates and subsequently result in survival benefits for those experiencing OHCA worldwide.

\section{Limitation}

This retrospective study was conducted with data from the IGOGO database; as a result, it has some limitations. First, we evaluated the performance in an educational setting; the current results may not represent children's actual performance when facing a real emergency situation. Finally, this 
study did not track the learning status; hence, we could not assess whether children's CPR abilities are maintained over time; this aspect may be considered in future research.

\section{Conclusion}

In summary, we revealed that sixth-grade elementary students' performance in CPR and AED was similar to that of adults after completing the current 90-minute course. Because children are more willing to perform CPR than adults are, we strongly advocate offering CPR and AED courses to 12 -year-old children, and these courses should emphasize checking the victim's breathing status.

\section{Data Availability}

The data that support the findings of this study are available from the corresponding author upon reasonable request (e-mail: ngowl@ms3.hinet.net).

\section{Conflicts of Interest}

The authors declare that they have no conflicts of interest.

\section{Authors' Contributions}

Ming-Fang Wang and $\mathrm{Yi}-\mathrm{Kan} \mathrm{Wu}$ contributed equally to this work. Cheng-Yu Chien and Chip-Jin Ng contributed equally to this work.

\section{Acknowledgments}

The authors would like to thank Wallace Academic Editing for English language editing. This research was supported by Chang Gung Memorial Hospital, Taiwan (CMRPG3K1251).

\section{Supplementary Materials}

CPR willingness questionnaire. (Supplementary Materials)

\section{References}

[1] G. Nichol, E. Thomas, C. W. Callaway, J. Hedges, J. L. Powell, and T. P. Aufderheide, "Regional variation in out-of-hospital cardiac arrest incidence and outcome," JAMA, vol. 300, no. 12, pp. 1423-1431, 2008.

[2] C.-Y. Wang, J.-Y. Wang, N.-C. Teng et al., "The secular trends in the incidence rate and outcomes of out-of-hospital cardiac arrest in taiwan-A nationwide population-based study," PLoS One, vol. 10, no. 4, Article ID e0122675, 2015.

[3] E. Marijon, W. Bougouin, M. Tafflet et al., "Population movement and sudden cardiac arrest location," Circulation, vol. 131, no. 18, pp. 1546-1554, 2015.

[4] C. Y. Chien, S. L. Tsai, L. H. Tsai et al., "Impact of transport time and cardiac arrest centers on the neurological outcome after out-of-hospital cardiac arrest: a retrospective cohort study," Journal of the American Heart Association, vol. 9, no. 11, Article ID e015544, 2020.

[5] C. Sasson, M. A. M. Rogers, J. Dahl, and A. L. Kellermann, "Predictors of survival from out-of-hospital cardiac arrest," Circulation: Cardiovascular Quality and Outcomes, vol. 3, no. 1, pp. 63-81, 2010.
[6] C. Sasson, D. J. Magid, P. Chan et al., "Association of neighborhood characteristics with bystander-initiated CPR," New England Journal of Medicine, vol. 367, no. 17, pp. 1607-1615, 2012.

[7] C. Sasson, C. C. Keirns, D. M. Smith et al., "Examining the contextual effects of neighborhood on out-of-hospital cardiac arrest and the provision of bystander cardiopulmonary resuscitation," Resuscitation, vol. 82, no. 6, pp. 674-679, 2011.

[8] M. J. Mitchell, B. A. Stubbs, and M. S. Eisenberg, "Socioeconomic status is associated with provision of bystander cardiopulmonary resuscitation," Prehospital Emergency Care, vol. 13, no. 4, pp. 478-486, 2009.

[9] L. B. Becker, B. H. Han, P. M. Meyer et al., "Racial differences in the incidence of cardiac arrest and subsequent survival," New England Journal of Medicine, vol. 329, no. 9, pp. 600-606, 1993.

[10] M. WissenbergF. K. Lippert et al., "Association of national initiatives to improve cardiac arrest management with rates of bystander intervention and patient survival after out-ofhospital cardiac arrest," JAMA, vol. 310, no. 13, pp. 1377-1384, 2013.

[11] C.-W. Kuo, L.-C. See, H.-T. Tu, and J.-C. Chen, "Adult out-ofhospital cardiac arrest based on chain of survival in taoyuan county, Northern Taiwan," The Journal of Emergency Medicine, vol. 46, no. 6, pp. 782-790, 2014.

[12] P. S. Chan, B. McNally, F. Tang, A. Kellermann, and C. S. Group, "Recent trends in survival from out-of-hospital cardiac arrest in the United States," Circulation, vol. 130, no. 21, pp. 1876-1882, 2014.

[13] E. Pei-Chuan Huang, W.-C. Chiang, M.-J. Hsieh et al., "Public knowledge, attitudes and willingness regarding bystander cardiopulmonary resuscitation: a nationwide survey in Taiwan," Journal of the Formosan Medical Association, vol. 118, no. 2, pp. 572-581, 2019.

[14] C.-Y. Chien, S.-Y. Fang, L.-H. Tsai et al., "Traditional versus blended CPR training program: a randomized controlled noninferiority study," Scientific Reports, vol. 10, no. 1, p. 10032, 2020.

[15] B. W. Böttiger and H. Van Aken, "Kids save lives," Resuscitation, vol. 94, pp. A5-A7, 2015.

[16] C.-C. Lin, C.-W. Kuo, C.-J. Ng, W.-C. Li, Y.-M. Weng, and J.-C. Chen, "Rescuer factors predict high-quality CPR-a manikin-based study of health care providers," The American Journal of Emergency Medicine, vol. 34, no. 1, pp. 20-24, 2016.

[17] S.-C. Hsu, C.-W. Kuo, Y.-M. Weng, C.-C. Lin, and J.-C. Chen, "The effectiveness of teaching chest compression first in a standardized public cardiopulmonary resuscitation training program," Medicine, vol. 98, no. 13, Article ID e14418, 2019.

[18] B. Banfai, E. Pek, A. Pandur, H. Csonka, and J. Betlehem, "“The year of first aid": effectiveness of a 3-day first aid programme for 7-14-year-old primary school children," Emergency Medicine Journal, vol. 34, no. 8, pp. 526-532, 2017.

[19] I. Jones, R. Whitfield, M. Colquhoun, D. Chamberlain, N. Vetter, and R. Newcombe, "At what age can schoolchildren provide effective chest compressions? an observational study from the Heartstart UK schools training programme," BMJ, vol. 334, no. 7605, p. 1201, 2007.

[20] D. M. Cave, T. P. Aufderheide, J. Beeson et al., "Importance and implementation of training in cardiopulmonary resuscitation and automated external defibrillation in schools," Circulation, vol. 123, no. 6, pp. 691-706, 2011.

[21] N. Plant and K. Taylor, "How best to teach CPR to schoolchildren: a systematic review," Resuscitation, vol. 84, no. 4, pp. 415-421, 2013. 
[22] A. Bohn, H. K. Van Aken, T. Möllhoff et al., "Teaching resuscitation in schools: annual tuition by trained teachers is effective starting at age 10. A four-year prospective cohort study," Resuscitation, vol. 83, no. 5, pp. 619-625, 2012.

[23] R.-P. Lukas, H. Van Aken, T. Mölhoff et al., "Kids save lives: a six-year longitudinal study of schoolchildren learning cardiopulmonary resuscitation: who should do the teaching and will the effects last?" Resuscitation, vol. 101, pp. 35-40, 2016.

[24] S. Calicchia, G. Cangiano, S. Capanna, M. De Rosa, and B. Papaleo, "Teaching life-saving manoeuvres in primary school," BioMed Research International, vol. 2016, p. 1, 2016.

[25] E. De Buck, H. Van Remoortel, T. Dieltjens et al., "Evidencebased educational pathway for the integration of first aid training in school curricula," Resuscitation, vol. 94, pp. 8-22, 2015.

[26] Education, Implementation, and Teams: 2020, "International consensus on cardiopulmonary resuscitation and emergency cardiovascular care science with treatment recommendations," Circulation, vol. 142, no. 16, pp. S222-S283, 2020.

[27] M.-F. Tsai, L.-H. Wang, M.-S. Lin, and M.-Y. Chen, "What do adolescents learn from a 50 minute cardiopulmonary resuscitation/automated external defibrillator education in a rural area: a pre-post design," International Journal of Environmental Research and Public Health, vol. 16, no. 6, p. 1053, 2019.

[28] R. Swor, I. Khan, R. Domeier, L. Honeycutt, K. Chu, and S. Compton, "CPR training and CPR performance: do CPRtrained bystanders perform CPR?" Academic Emergency Medicine, vol. 13, no. 6, pp. 596-601, 2006.

[29] H. H. Chen, W. C. Chiang, M. J. Hsieh, C. H. Lee, Z. F. Yuan, and H. Y. Lin, "Experiences and psychological influences in layrescuers performaing bystander cardiopulmonary resuscitation:a qualitative study," Journal of Acute Medicine, vol. 10, no. 4, pp. 138-148, 2020. 